\title{
Astrocytomas children grade ii in frontal region case study
}

\begin{abstract}
Infantile astrocytoma is characterized by its low degree of malignancy, are cellular tumors of heterogeneous trait, usually presented little delimited and infiltrative without signs of anaplasias. The reported case is a child of 05 (five) years, female with diagnosis of grade II astrocytoma with clinical symptoms of lower extremity hypotonia and upper and lower limb hypotrophy without facial mimicry, low weight and difficulty in performing typical movements of age. The purpose of this study is to identify the best physiotherapeutic resources for this patient, as well as to describe the real gains during therapy through the insertion of a protocol of care, neuropsychomotor development due to astrocytoma. The physiotherapeutic intervention in these cases shows that the insertion of a care protocol according to the patient's clinical condition was effective from the point of view that allowed the patient to try new movements, to harmonize the existing ones and to enable them to be inserted into everyday activities within the scope familiar and ensuring action in the social context.
\end{abstract}

Keywords: astrocytoma, central nervous system neoplasms
Volume 9 Issue 2 - 2018

\author{
Carla Chiste Tomazoli Santos,' Cristilene \\ Akiko Kimura, ${ }^{2}$ Chadya Samia Soares \\ Pacondes De Miranda, ${ }^{3}$ Kelly De Jesus \\ Menezzes Da Silva, ${ }^{4}$ Amanda Cabral dos \\ Santos, ${ }^{5}$ Fellipe José Gomes Queiroz ${ }^{6}$ \\ 'Physioterapist, Master in Physiotherapy, Faculty of Sciences and \\ Education Sena Aires(FACESA), Brazil \\ ${ }^{2}$ Nurse, PhD in Nursing, Faculty of Sciences and Education Sena \\ Aires(FACESA), Brazil \\ ${ }^{3}$ Physiotherapy students, Faculty of Sciences and Education Sena \\ Aires(FACESA), Brazil \\ ${ }^{4}$ Physiotherapy students, Faculty of Sciences and Education Sena \\ Aires(FACESA), Brazil \\ ${ }^{5}$ Physiotherapist, Master in physiotherapy, Faculty of Sciences \\ and Education Sena Aires, Valparaíso de Goiás, Brazil \\ ${ }^{6}$ Pharmacist, Master in Pharmaceutic Sciences, Faculty of \\ Sciences and Education Sena Aires, Valparaíso de Goiás, Brazil
}

\author{
Correspondence: Carla Chiste Tomazoli Santos, Master \\ in Physiotherapy, Faculty of Sciences and Education Sena \\ Aires(FACESA), Acre St., Square 2- Sector of Chácras \\ Anhanguera,Valparaíso de Goiás - GO, Brazil, Zip: 72870-508, \\ Tel +55 6I 3627-4200, Email carlachiste@senaaires.com.br
}

Received: March 28, 2018 | Published: April 17, 2018

\section{Introduction}

Brain tumors of astrocytic origin are the most common form of childhood neoplasms, responsible for approximately $20 \%$ of intracranial tumors of this age group. They can also be known as gliomas, which refer to tumors originating in glial cells (oligodendrocytes, astrocytes, microglia and ependymas) and classified as grade I, less aggressive, and grades II, III and IV, thus varying their malignancy content. Malignant neoplasms are the second leading cause of death in children under 15 years of age, behind only death from accidents. Gliomas constitute a considerable and possibly the most important group of intracranial tumors, have a high level of complexity due to the numerous cellular types involved and can be subdivided into diffuse or focal.

Grade II astrocytomas have an exacerbated differentiation behavior, their histological character is characterized by homogeneous masses with irregular borders, which occur due to a probable mutation of the p53 gene (inducing hereditary predisposition to cancer) and loss of heterozygosity (different alleles for a given gene ) of chromosome 17. In Brazil, about 1,500 to 2,000 new cases of gliomas are expected every year. The etiology of these brain tumors does not have a specific cause, but there are indications that they describe a greater incidence when they are related to some types of syndromes. ${ }^{1}$

The classification of $\mathrm{OM}$ represents a scale of malignancy, considering the clinical and biological behavior of the neoplasms, the genetic profile and the prognosis of the tumors, influencing the choice of therapies, including radiotherapy and chemotherapy protocols. ${ }^{2}$
The clinical picture is variable and depends especially on its location; as a rule, most central nervous system neoplasms present with symptoms such as headache (variable or localized or diffuse pain in any part of the head), signs of intracranial hypertension blood pressure), cerebellar ataxia (difficulty in coordinating body movements), pyramidal signs (a set of signs and symptoms they reach the periphery), loss of balance, nausea, anorexia and lethargy, the onset and evolution are insidious and slow. ${ }^{2}$

The diagnosis is made from a computerized tomography (CT), with thin sections $(5 \mathrm{~mm})$ for better visualization, it is also possible to use magnetic resonance imaging (MRI), which is more sensitive to evaluate structures such as temporal lobe and posterior fossa, besides not exposing the child to radiation, are tests of paramount importance for the surgical decision. ${ }^{3}$

The conventional treatment for this type of tumor is based on complete surgical removal of the tumor mass, associated to the use of chemotherapy and later radiotherapy. ${ }^{3}$

\section{Materials and methods}

Participated in this study a family composed of father, mother and child, separated parents but living in the city of Valparaíso de Goiás, the child is female, with 5 years. The data collection was done by the researchers in the clinic school of the Faculty Facesa in Valparaíso de Goiás under the supervision and supervision of the Master Teacher Carla Chiste Tomazoli Santos, with weekly consultations lasting one hour for a period of six months. Two techniques were used to collect 
data: questionnaire, semi-structured interviews with direct observation of behavior, using the resources of video and photo technology. Firstly, the questionnaire was applied to the mother with the purpose of characterizing and knowing the family. Information on age and schooling, occupation and marital status of the parents, social contacts of the family and identification of those responsible for the routine tasks of the child were collected. The interviews were performed with the mother and the father, separately, and recorded in a report on the patient's chart. The interview addressed the following themes: a) life history of the child: pregnancy, childbirth, neuropsychomotor development, sociability, schooling and diseases; b) information about the diagnosis of the child: initiatives taken and reaction of the parents to the diagnosis; c) information about the habits and customs of the child and the family; d) behavioral and emotional characteristics of the child; and e) expectations of the parents regarding the child with infant astrocytoma. The interview with the father of the child included questions about the role played by him in the family context, besides the subjects investigated in the interview with the mother.

\section{Results and discussion}

This chapter will address a case study with a 5-year-old female patient from an unplanned pregnancy. The mother had the following gestational complications: urinary tract infection, headache (pain of varying intensity, localized or diffuse in any part of the head), and hypertension (high blood pressure in the arteries).

The mother had completed a full prenatal care, fulfilling all the criteria given by the obstetrician at that time, the child was born in a normal hospital delivery (PNH) with 37 weeks, weight of 2,940 g length of $46 \mathrm{~cm}$ and cephalic circumference of $34 \mathrm{~cm}$. Late.

In the first moments of the patient's life, a generalized cyanosis and APGAR (The APGAR Scale or Index consists of the evaluation by a pediatrician of 5 objective signs of the newborn, with each of the signs being assigned a score of 0 to 2) with a score of 2 at birth, thus requiring an emergency ventilatory support that lasted approximately six (06) hours and was referred to the room after this period.

The child was diagnosed with astrocytoma GII of frontal region with involvement of the corpus callosum in the year 2011, with resection of $90 \%$ in 2014 and new approach in 2015 with good response to chemotherapy. He started attending at the physiotherapy clinic in the Faculty of Sena Aires with a functional kinetic frame: lower limb hypotonia and upper and lower limb hypotrophy, not performing dorsal decubitus changes to sedestation and vice versa, rolling in block when asked to return the initial position does dissociation of the pelvic and scapular girdle, rectifying cervical reaction and rectification body reaction present, anterior, posterior and lateral protection reactions in development, does not perform papping and semi papping, under inspection presents trigonocephaly, face expressionless, thorax in with a central catheter in the right hemithorax, knees, ankles and valgus feet, makes use of ankle and foot orthoses (AFO) to walk using the $\mathrm{AFO}$ orthosis with the aid of the walker and under stabilization of the hip key point.

During the genetic examination, the patient suffered a cardiorespiratory arrest remaining in the intensive care unit for five (05) months due to the post-stop complications. The result of the genetic test was presented without alterations, thus excluding any type of syndrome. When she was discharged from the ICU, the patient was referred to a pediatric unit bed where she remained in use of Oxygen therapy and a nasogastric tube for feeding.
The child was diagnosed with astrocytoma GII of frontal region with involvement of the corpus callosum in the year 2011, with resection of $90 \%$ in 2014 and new approach in 2015 with good response to chemotherapy. He started attending at the physiotherapy clinic in the Faculty of Sena Aires with a functional kinetic frame: lower limb hypotonia and upper and lower limb hypotrophy, not performing dorsal decubitus changes to sedestation, rolling in block when asked to return the initial position does dissociation of the pelvic and scapular girdle, rectifying cervical reaction and rectification body reaction present, anterior, posterior and lateral protection reactions in development, does not perform papping and semi papping, under inspection presents trigonocephaly, face expressionless, thorax in with a central catheter in the right hemithorax, knees, ankles and valgus feet, makes use of ankle and foot orthoses (AFO) to walk using the AFO orthosis with the aid of the walker and under stabilization of the hip key point.

The treatment is based on motor activities and sensory stimulation, with the aim of facilitating neuropsychomotor development, adjusting tonus, promoting muscular strength gain, playful transfer training and protective reactions, stimulating gait with device evolving to gait without auxiliary device and promoting proprioception and interaction with facial and body expression besides inhibiting pathological patterns.

The stimulation and encouragement of the typical movements of the age group provide the integration of the patient in their daily activities and guarantees an improvement in their physical and social and emotional state.

The major challenge in this patient is the maintenance, support and harmonization of the children's movement, for this we aim the planing and holding (placement and maintenance) with the objective of facilitating the normal motor development, promoting a selfpostural adjustment and the maintenance of the same without aid of the therapist, the use of tapping, which aims to increase the postural tone by the tactile and proprioceptive stimulus in certain key points, helps to activate weak muscle groups, stimulates balance reactions and equilibrium movements that develop and execute more synergistic and stable movements by co-contraction avoiding pathological patterns. The tapping used in the patient of this case is that of pressure. ${ }^{4,5}$

Already for the maintenance of range of motion, muscular strength gain and proprioceptive training, an infant bicycle was used, with the stabilization of the feet on the pedals without the use of the AFO orthosis, in order to promote a new experience with a different texture, besides to provide moments of autonomy by letting it define the path we are going to go through.

Music therapy has been a great ally, with the definition of musical tastes used the backrest to train the sit and raise always dancing and singing in order to make that moment pleasant, stimulating new posture arrangements.

Jokes such as bowling and back-to-back toy with different ball weights were sought to increase the strength of the upper limbs, seeking harmony between the limbs and facilitating fine motor activities.Face painting and jokes to encourage facial and postural expression reactions.

Martial arts training with tales of super heroines to achieve motor autonomy and encouraging motor learning, experiencing new movements and postures. 


\section{Final considerations}

The importance of physical therapy in these cases is the promotion of quality of life, through the facilitation of typical movements to age, prevention of pathological patterns, promotion of functional independence, and insertion into the child and school environment, encouraging physical, social and emotional well-being. The functionality of the motor acquisitions are of paramount importance because they guarantee independence to the patient, stimulating autonomy in daily activities, physiotherapy through a holistic approach intervene in the motor response of children with low grade or grade II astrocytoma who present lesions in some system, reorganizing functional movements, and encouraging the relearning motor.

\section{Acknowledgement}

None.

\section{Conflict of interest}

Authors decare there is no conflicts in publishing the article.

\section{References}

1. SilvaLC. Immunohistochemical and Molecular Study of Gliomas Belo Horizonte. Federal University of Minas Gerais Faculty of Medicine. 2014.

2. Malheiros SMF, Stávale JN, Franco CMR, et al. Diffuse Astrocytomas of Low Degree of Malignancy. Rev Neurosciences. 1998;6(2):75-80.

3. Barros JM. Low grade diffuse astrocytomas. Marco de Canaveses Portugal. Faculty of Medicine, University of Porto. 2011.

4. Negreiros AA, MonteiroLL, Arruda RF, et al. Epidemiological Aspects of Pediatric Central Nervous System Tumors in a Reference Hospital of João Pessoa (PB) between 2009 and 2011. 2015;M\&PN ${ }^{\circ}$ 1:51-59.

5. Peres LW, Ruedell AM, DiamanteC. Influence of the Neuroevolutionary Bobathon Concept on Muscle Strength and Tonic Functionality Static and Dynamic Functions in Dysarthrophysitic Patients after Paralysis. Health, Santa Maria. 2009;35(1):28-33. 